\title{
THE TREATMENT OF GENERAL PARALYSIS OF THE INSANE BY MALARIA.*
}

\author{
By HENRY J. MACBRIDE, London, AND W. L. TEMPLETON, \\ Bristol.
}

THIs paper is offered as an addition to the recorded preliminary results of the treatment of general paralysis by malaria, and is based upon a series of eighteen cases.

\section{INTRODUCTION.}

It is of interest to note that the method was suggested by WagnerJauregg (quoted by Gerstmann ${ }^{1}$ ) as far back as 1887, but was not systematically employed by him till 1917, when nine cases of general paralysis were inoculated with benign tertian malaria. The experiments were interrupted ' in order to await results,' and were not resumed until September, 1919. The improvement in the nine cases was so striking that, since this latter date, all cases of general paralysis admitted to the Wagner-Jauregg Klinik have been treated by malaria as soon as the condition has been diagnosed.

The principle depends on the fact that in chronic disease remissions may occur after an attack of an acute specific fever. Many methods of producing fever artificially have been tried with varying results. Wagner-Jauregg, according to Pilcz, ${ }^{2}$ after a thorough investigation of the various methods employed, and of the cases of so-called 'spontaneous' remission, has drawn up a scale of efficiency as follows : (1) chemical substances, e.g., sodium nucleinate ; (2) toxalbumins, e.g., tuberculin and staphylococci ; (3) acute disease. The last group was found to be the most efficient.

The rationale of none of the various methods used is as yet known. In contrast to some, e.g., sodium nucleinate and 'phlogetan,' in which the essential factor seems to be a neutrophile leucocytosis, ${ }^{3}$ with or without pyrexia, it is to be emphasized that in malaria a leucopœnia and not a leucocytosis is the rule, and this has been confirmed in many of our cases. It has been suggested that the improvement obtained by virtue of intercurrent disease or of artificially produced fever is due simply to a quickening of metabolism with the removal of accumulated

* This paper was communicated to the Psychiatry Section, Royal Society of Medicine, London, on December 12, 1923. 
waste products. On the other hand, it may be that high temperatures exercise some destructive influence on the spirochætes in the body, or, again, that the destruction of the red blood corpuscles, followed by rapid regeneration from the hæmopoetic centres, may lead, as suggested by Muhlens and Kirschbaum, ${ }^{4}$ to a non-specific antibody production. The biological relationship between the plasmodium and the Spirochota pallida, both protozoa, must not be forgotten, and one might, indeed, formulate the hypothesis of a specific antibody production against protozoa. It is of interest to note in this connection that in a series of cases of dementia præcox treated by malaria (a full report of which will appear in a forthcoming publication), the mental improvement obtained was of a fleeting nature, in contrast to the more permanent and progressive results obtained in general paralysis; all of the dementia præcox subjects relapsed within three months.

\section{TECHNIQUE.}

As a method of producing fever, experiments have been made with benign tertian malaria, tropical malaria and relapsing fever. Of these three, benign tertian malaria was found to give the best results, no particular advantage being obtained from the higher temperatures of relapsing fever. ${ }^{1}$

The method employed in the Wagner-Jauregg Klinik, in Vienna, consists in the withdrawal of from 2 to 4 c.c. of blood from the vein of a patient suffering from benign tertian malaria and in its subcutaneous injection into the general paralytic. The site chosen is the area between the scapula and the spine. (It is not essential that the blood should be withdrawn during the febrile attack.) By this method, the incubation period varies from one to three weeks, rarely less. In most of our cases blood was injected intravenously, to avoid clotting. It is advisable to have the syringe lubricated with liquid paraffin. By this latter method the pyrexia developed in most cases on the second or third day, but ran a somewhat irregular course for a few days, and then began to show typical rises every second day, with rigors, etc. In some cases, after a varying period, twelve to sixteen days, daily rigors developed and continued. In a few cases, daily rigors occurred from the beginning, i.e., a quotidian fever developed, in this case double tertian. The advantages derived from quotidian fever, viz., that the course of treatment was accordingly shorter, and that the fever reactions were more or less continuous, were, in our opinion, outweighed by the fact that the patient was continuously prostrated.

Several strains of the malarial parasite have been used in the present series of cases (one strain has passed through ten different hosts), and we are of the opinion that the virulence tends to be increased with 
each successive transmission. This is in agreement with the findings of Grant, ${ }^{5}$ and we consider it advisable, therefore, to renew the strain at every available opportunity from fresh untreated cases of malaria.

The patient is allowed to have, on an average, twelve high-fever reactions, the actual numbers depending more or less on his state of health. The fever is terminated by the administration of quinine. Wagner-Jauregg ${ }^{6}$ suggests 15-grain doses each day for three days, and, during the fourteen following days, half that dose daily. In our experience the first dose of quinine is, in most cases, sufficient to terminate the fever entirely. It has been suggested that the sudden cessation of the fever on the administration of quinine in artificially-produced malaria is due to the absence of the more resistant sexual forms of the parasite.

Several cases failed entirely to develop fever, and a few recovered spontaneously without the administration of quinine. It is of interest to note that all of these patients had already suffered from malaria abroad, but there were others who, in spite of previous malarial infection, developed fever in the usual way after inoculation.

In all our cases, following upon the febrile attacks and running parallel with the quinine treatment, weekly doses of N.A.B. or salvarsan were administered intravenously, six doses in all being given. Though directed primarily against the syphilitic infection, these injections were remarkably efficacious in relieving the profound anæmia which followed upon the malarial paroxysms.

\section{COMPLICATIONS.}

During the course of the fever, epistaxis occurred in a few cases, and herpes febrilis was common. In a minority only was the spleen palpable. Acute retention of urine was a troublesome complication. Three cases had quite severe vomiting at the height of the individual fever reactions. Anæmia, of course, was profound in all of the cases, but recovery was rapid on the termination of the fever. Unfortunately we have to report three deaths which occurred during the course of the treatment. Other observers have reported a considerable number of deaths, but give no details. Gerstmann, ${ }^{1}$ commenting on twelve fatal cases, states simply that in none did the post-mortem examination reveal any evidence of death from malarial infection. Grant, ${ }^{5}$ quoting six deaths in forty cases, does not state at what stage of the treatment these occurred. The details of our three fatal cases are as follows :-

Case 1.-Male, aged thirty-five. Admitted October 6, 1923.

He had malaria in 1916, but otherwise enjoyed good health till about one year before admission, when he became very irritable and hyperexcitable. Nine months later he had a seizure, but felt quite well 
the following day. After an interval of two months he had two seizures within half an hour of each other, in which he bit his tongue and exhibited incontinence of urine. During the few months before admission the patient often fell down owing to a sudden giving way of his legs, but there was no loss of consciousness.

On examination no abnormality was found in the cardiovascular, respiratory, alimentary or genito-urinary systems. The pupils reacted well to light; tremors were present in the lips and tongue; dysarthria was marked; there was widespread diminution to painful stimuli; motor power was good; tendon reflexes were absent; plantars both flexor ; sphincters normal ; Rombergism slightly positive ; Wassermann reaction strongly positive in the blood and cerebrospinal fluid.

Mentally the patient was able to give a fair account of himself, and had a fair insight into his condition, but his memory was poor; he was disorientated for time and place, and was emotionally unstable.

He was inoculated with 2 c.c. benign tertian malarial blood intravenously, on October 13. Six hours after, he had a seizure and his temperature rose to $107^{\circ} \mathrm{F}$., when he immediately went into a status epilepticus. From this he passed into a semicomatose condition and died on October 19, in spite of intravenous quinine medication, etc.

Case 2.-Male, aged thirty-three. Admitted July 24, 1923.

Patient complained of loss of memory. His wife stated that for the last year he had been mentally confused, and had an attack several months before admission, in which he lost his speech for twenty minutes, without loss of consciousness.

On examination, the cardiovascular, respiratory, alimentary and genito-urinary systems appeared normal. The pupils were unequal and did not react to light, but did on accommodation; pinprick was diminished generally; motor power was good; knee jerks absent; plantars both flexor; sphincters normal ; Rombergism slight.

Mentally he was very confused and childish; memory bad; emotionally very unstable; he gave an unsatisfactory account of himself and was a very poor witness; the Wassermann reaction was strongly positive in the blood and cerebrospinal fluid.

On August 7 the patient was inoculated intravenously with benign tertian malaria. After a slightly irregular febrile reaction the true fever developed on October 12. From this time the patient's mental condition deteriorated with each daily rise of temperature. He refused to take sufficient nourishment, and was with difficulty fed per rectum. On October 18, an attempt was made to stop the malaria, on account of the progressive prostration of the patient, but he died on October 21 .

Case 12.-Female, aged forty-two. Admitted August 8, 1923.

During the three years previous to admission, the patient had been having progressive difficulty in her speech and was losing her memory. 
Walking was becoming difficult during the same period on account of weakness in the legs.

On examination, the cardiovascular, respiratory, alimentary and genito-urinary systems appeared normal. The pupils were unequal, and only the right reacted to light; face and tongue were tremulous ; dysarthria well marked; motor power fairly good; outstretched hands coarsely tremulous ; knee-jerks brisk ; plantars both flexor ; sphincters normal; the Wassermann reaction was strongly positive in the blood and cerebrospinal fluid.

Mentally the patient was childish and completely disorientated for time and place, with defective memory.

An intravenous injection of about 3 c.c. of benign tertian malarial blood was given on August 7. A reaction occurred the following day, the patient's temperature rising to $102^{\circ} \mathrm{F}$. by the evening. The malarial rigors began on August 12, though for the previous five days the temperature had been slightly raised in the evening. Ten daily rigors occurred, and the patient died after the tenth, in spite of the administration of intravenous quinine, both during the first rigor and afterwards.

During the period of treatment, the patient emaciated rapidly. The trembling in the hands, noted previously, became more marked. The movements became jactatations, the finger and arm jerkings resembling the terminal clonic movements of an epileptic seizure. During the rigors, there was considerable cyanosis. The spleen was palpable.

Post-mortem examinations were made in two of the cases, and in these, evidence of marked splenic enlargement, with bile staining of the liver substance, was found. It may be admitted, then, that patients died of malarial infection.

No autopsy was obtained in the case which died in a condition of status epilepticus. It may be that the intravenous injection of a foreign serum caused a certain degree of protein shock, sufficient to send the patient into this state. It is to be noted that there was a history of seizures prior to admission.

\section{RESULTS RECORDED BY OTHERS.}

Of 350 cases treated in the Wagner-Jauregg Klinik down to February, 1923, ${ }^{7}$ complete observations were made in 296 only. Of these no fewer than 202 showed remissions of varying degree, while 112 showed complete remission with the disappearance of the former mental symptoms and a return of the former business capacity; remissions were noted in 68 per cent., and complete remissions in 36 per cent. A recent (personal) letter from Dr. Gerstmann states that treatment is voL. v.- vo. 17. 
being continued in the Wagner-Jauregg Klinik, with a very similar proportion of remissions.

Of the patients who had complete remissions, three treated in 1917 are still actively employed at business and show no sign of relapsing. These remissions have, therefore, been of six years' duration. In seventeen cases the remissions have already lasted two to three years and, in a further thirty-four, one or two years. Of the total cases of complete remission, three only have relapsed or shown any tendency to relapse.

Many of the advanced cases of the disease gave only very incomplete remissions, but even in those much benefit was obtained, particularly in the cessation of acute symptoms and in the non-progression or even regression of the dementia. Of those patients already in a profound state of dementia, many again became sociable and inoffensive, and regained a certain degree of occupational ability.

The remissions do not follow immediately upon the febrile attacks ; Gerstmann states that the first sign of improvement may not be visible for weeks or months after the termination of the fever. One must, therefore, be especially careful not to come to any definite conclusion until sufficient time has elapsed. The course of remission is held to be progressive, without further inoculation, though this may be necessary in cases which remain stationary, say after an interval of six to twelve months. Some cases which showed an incomplete remission, when re-examined months later, showed a full and complete return to the normal.

There is apparently no parallelism existing between the clinical and serological findings. Many cases with complete remission of symptoms have still a positive Wassermann reaction in the blood and cerebrospinal fluid; a few, examined months later, have shown a negative result in both. It is found that the blood becomes negative before the cerebrospinal fluid, and that improvement is first seen in a reduction of the number of cells in the fluid. To bear out this absence of parallelism, Gerstmann quotes a case successfully treated by the tuberculin-mercury method in 1909, in which there has been no relapse. When this patient was examined recently, the report was as follows :-

Wassermann reaction positive in the blood and cerebrospinal fluid, globulin test positive, cells 186 .

Case 16 of our series, one of non-progressive general paralysis, is of interest in this connection.

Male, aged forty-eight. Admitted March 9, 1916.

The disease was of eighteen months' duration on admission.

On examination, the general systems showed nothing abnormal. Nervous system : pupils unequal and Argyll Robertson in type ; speech slurred ; hands showed a coarse tremor ; knee jerks brisk ; plantars, R., 
extensor, L., flexor; Wassermann test positive in the cerebrospinal fluid and blood. Mentally the patient was exalted and had grandiose ideas.

July, 1923. The patient was weak-minded and childish, irritable, asocial and occasionally faulty in his habits. For about a year his state had been apparently quiescent. Just prior to his inoculation with malaria, his blood and cerebrospinal fluid were again examined and found to be completely negative. In spite of this, however, the induction of malaria was proceeded with, and he had twelve rises of temperature above $103^{\circ} \mathrm{F}$. During the fever paroxysms he improved mentally, became more agreeable, more rational in conversation, and clean in his habits. This persisted for about six weeks only, and three months later he had completely relapsed.

According to the Vienna report ${ }^{1}$ the results of treatment depend essentially on the stage of the disease - the earlier the stage, the more certain the remission. In cases of short duration Wagner-Jauregg claims that entire success can be predicted with almost absolute certainty. According to Gerstmann, the types of case giving the best results are those of simple dementia and of taboparesis; Pilcz ${ }^{2}$ adds to this the cases with maniacal symptoms.

The development of acute mental symptoms during the febrile stage is regarded as a favourable omen, though in some cases they persist after the fever has terminated. This is especially true of auditory hallucinations, which may persist in spite of an otherwise complete remission.

The importance of an anatomical control of clinical remissions is obvious. Gerstmann ${ }^{8}$ quotes the histological findings in three cases which had shown complete remissions, the patients dying later of intercurrent disease. The changes were so poorly developed, compared with the customary findings, that without knowledge of the cases the anatomical diagnosis would have caused some difficulty. He comments upon the greater infiltration of the temporal lobes, as compared with other certical regions, and suggests a possible connection between this fact and the frequent occurrence and persistence of auditory hallucinations.

Scripture, ${ }^{9}$ in a survey of the recent results from the same clinic, states that, during a year and a half, of 141 cases treated, sixty-two were - completely cured. These, he says, were the early cases, in which the disease had not been present for more than two and a half years.

Most of the German clinics have already made use of the method, with apparently good results. Weygandt ${ }^{10}$ reported fifty cases, with remission in 88 per cent., and good remission in 48 per cent. Kirschbaum, ${ }^{11}$ in a series of fifty-one cases, obtained $58 \cdot 8$ per cent. good remissions, and 13.7 per cent. remissions of lesser degree-in all, 
remission in $66 \cdot 6$ per cent. This same investigator, from a survey of cases admitted prior to the introduction of malaria therapy, showed that on the average 'spontaneous' remissions accounted for no more than 11.4 per cent. of the total. In this connection Robertson ${ }^{12}$ has called attention to the remarkable decrease in the number of deaths from general paralysis within recent years, and this must be remembered in arguing from statistics as to the beneficial results of any form of treatment.

McAlister, ${ }^{13}$ reporting recently on a series of twelve cases treated at Morningside, concluded that (1) of nine cases, two only were conspicuously improved, in one the emphasis being on the mental side, and in the other on the physical ; (2) four other cases showed some improvement, although not so marked. The average duration of the disease in his twelve cases was two years, and yet, as the author says, "there is a complete absence of what, even when the term is generally interpreted, might be described as 'cures." He discusses the question whether the infection with malaria exerts any particular influence upon the permeability of the choroid plexus to salvarsan.

Gerstmann, ${ }^{14}$ however, does not consider that subsequent treatment with neosalvarsan is at all essential ; in fact, it has been repeatedly neglected for various reasons, without disadvantage.

Grant ${ }^{5}$ has recorded his preliminary observations in a series of forty cases treated during the past fourteen months. Of these, three patients have been discharged to their homes and are now following their usual occupation. Three, formerly 'wet and dirty' in their habits, have ceased to be so. Two patients, formerly confined to bed, have regained their strength to a considerable degree, and are able to be up and about.

\section{PERSONAL CASES.}

Of the present series of eighteen cases, subjected to the malarial treatment, two were cases of juvenile general paralysis. Of the remaining sixteen, eleven may be classified as early cases, the average duration of the symptoms being of ten months only ; in five, the duration on an average was four years.

I. EARly Cases. - Cases 1 and 2 of this series have already been referred to as having had a fatal issue during the course of the treatment. 1923.

Case 3. - A fairly robust male, aged forty-eight. Admitted July,

He had complained of shooting pains in the left arm and leg for some nine months. A fortnight before admission he collapsed while walking upstairs, but was not unconscious. His wife stated that his disposition had altered lately.

On examination, his general systems appeared normal. Nervous 
system : pupils unequal and Argyll Robertson in type ; twitching of the face and fine tremors of the tongue ; dysarthria ; diminution to pinprick on the left side ; reflexes normal ; Wassermann reaction strongly positive in the cerebrospinal fluid.

Mentally he was definitely grandiose in a childish way; memory was poor.

August 8. The patient was inoculated with malaria and allowed to have twelve rises of temperature. The reaction was severe and the spleen became palpable. After the first dose of quinine the temperature did not rise again.

November 15. The patient was a 'new man' physically. He was stronger, and his weight was $20 \mathrm{lbs}$. heavier than before the treatment; speech was more normal ; tremors not so marked; memory improved. He was still mildly grandiose.

Case 4.-Male, aged fifty-three. Admitted August 9, 1923.

For about eighteen months there had been progressive weakness in his limbs with trembling; speech had been defective for some months. He had had malaria about twenty years ago.

On examination the general systems showed nothing abnormal except for some signs of chronic bronchitis. Nervous system : pupils equal, and of Argyll Robertson type; tongue and lips tremulous; dysarthria present; power in the legs slightly diminished ; knee jerks, R., brisk, L., present ; ankle jerks both absent; plantars both flexor ; difficulty in bladder control; Rombergism present.

On August 25 the patient was inoculated with malaria, the first rigor appearing on September 1. He was allowed to have twelve fever reactions, and after the first quinine dose the temperature remained normal. Nearly a stone in weight was lost during the treatment, but this was shortly afterwards regained.

Two months after, the patient was less tremulous and his speech had improved.

Case 5.-Male, aged fifty-four. Admitted August 9, 1923.

For several months before admission the patient found difficulty in doing his work, which entailed a great deal of writing. During this time his speech had become defective and his memory impaired.

On examination the general systems appeared normal. Nervous system : pupils reacted to light and were equal ; motor power good; no sensory loss ; knee jerks brisk; plantars both flexor ; occasional incontinence of urine.

He could not give a very good account of himself and was slightly confused ; memory defective ; dysarthria present.

Inoculation with malaria was started on September 14, the first rigor occurring on September 19. The fever remained true to type till September 28, when the rigors became a daily occurrence. After twelve 
rigors these were stopped with quinine. During the fever, towards the end of the treatment, the patient became maniacal with each rise of temperature, was doubly incontinent, and had visual hallucinations. This all disappeared with the cessation of the fever.

Two months later the patient was discharged as being ' very well.' His speech and memory were good, and he had become mentally clear. His physical condition was good and still improving. Writing had become normal again. At present he is convalescing at the seaside before resuming his work.

Case 6.-Male, aged forty-two. Admitted June 29, 1923.

There was a history of a slight seizure about four months before admission, otherwise the patient felt perfectly well.

On examination his general systems appeared normal. Nervous system : pupils Argyll Robertson in type ; reflexes normal ; sphincters normal ; motor power good; Wassermann reaction strongly positive in the blood and cerebrospinal fluid.

He showed marked euphoria and was very loquacious; there was no insight into his condition ; speech fairly good; no delusions.

On July 9 he was inoculated with malaria, and almost from the day of injection he had daily rigors. After twelve reactions quinine was given, when the rigors ceased. He vomited thrice and developed retention of urine, but otherwise there were no complications.

About two weeks after the termination of the fever, he developed marked grandiose ideas and, shortly after, hallucinations. His mental condition became such that he had to be sent to an asylum.

Case 7.-Male, aged fifty. Admitted July 11, 1923.

For several months before admission the patient's manner had been changing, and he had been having difficulty in controlling his bladder.

On examination the general systems appeared normal. Nervous system : pupils equal, L. reacting to light, while the R. was immobile, but both reacted on accommodation; dysarthria was present ; motor power good ; reflexes normal ; sphincters, slight incontinence of urine ; Wassermann reaction strongly positive in the blood and cerebrospinal fluid.

He was euphoric and had poor insight into his condition; memory poor ; slightly disorientated.

On July 19 he was inoculated with malaria and was allowed to have twelve fever reactions, when quinine was given. During the fever there was marked retention of urine.

For about two weeks after the treatment the patient's mental contion improved slightly and his speech seemed better, but cystitis set in and the patient ultimately died on November 5 .

Case 8.-Male, aged fifty-six. Admitted July, 1923.

For over a year he had been unable to lo ok after himself properly, and 
had marked delusions and hallucinations. He was very troubled with insomnia.

On examination no physical signs could be found, except Argyll Robertson pupils; the Wassermann reaction was strongly positive in the blood and cerebrospinal fluid. Mentally he showed marked euphoria and had no insight into his condition ; speech was fairly good ; memory defective, with well-marked delusions and hallucinations.

He was inoculated with malaria on July 19, and was allowed to have twelve fever reactions, when the fever was terminated with quinine. During the treatment he slept better than he had done for months, and no hypnotics were required.

Four months after, he was a 'new man' in many ways. He was physically very strong; mentally he was not confused and could do business transactions under supervision; the delusions and hallucinations were still mildly present. He was still making progress when he left hospital.

Case 9.-Female, aged fifty-three. Admitted February 1, 1923.

On examination the general systems appeared normal. Nervous system : pupils Argyll Robertson in type ; ataxic gait ; speech slurred ; Wassermann reaction strongly positive in the blood and cerebrospinal fluid.

Mentally she was exalted, with delusions of grandeur, supposing herself to be 'Queen of England,' etc. For four months after admission she remained in the same condition. Just prior to inoculation with malaria the patient began to lose some of her ideas of grandeur, but remained suspicious and querulous.

She was inoculated on June 26, and was allowed to have twelve fever reactions. On recovery from the effects of the fever, she became quite natural; her gait improved and she remained free from her delusions up to the date of her discharge in September. A later report states that the patient remains well.

Case 10.-Male, aged forty-seven. Admitted June 27, 1922.

The general systems were normal. Nervous system: double primary optic atrophy; tremors of the tongue and arms; ataxia; knee and ankle jerks absent; Wassermann reaction positive in the cerebrospinal fluid.

Mentally he had marked delusions of grandeur, with general exaltation and excitement. Up to the date of inoculation the patient remained exhilarated and deluded, but in a lesser degree than on admission. His memory was defective, and he did not know how long he had been in the institution.

On March 31 he was inoculated with malaria, and allowed to have seventeen rises of temperature above $103^{\circ} \mathrm{F}$. During the febrile stage he was unusually garrulous and quarrelsome, but on the termination of 
the fever this rapidly disappeared. He was then free from delusions, and his memory was very much improved. Physically, too, he was very much improved and was much steadier on his feet. The tremor of the arms had gone, though a slight tongue tremor remained. He was discharged on September 28.

Case 11.-Male, aged fifty-eight. Admitted September 29, 1922.

On examination the general systems appeared to be normal. Nervous system : pupils Argyll Robertson in type; tremors of the tongue, lips, arms and legs ; knee jerks brisk; plantars both flexor; Wassermann reaction strongly positive in the blood and cerebrospinal fluid.

Mentally the patient was exalted and aggressive, and had marked delusions of grandeur. Up to the date of inoculation he retained all his delusions and remained in the same physical state as on admission, with the addition of considerable loss of motor power in the legs.

On July 29, 1923, he was inoculated with malaria and was allowed to have eleven rises in temperature above $103^{\circ} \mathrm{F}$. During the course of treatment the patient became quite confused, but only on the days of fever, and on the termination of the attacks he made a rapid recovery.

During October his memory remained good; he was free from delusions and had a wonderful insight into his previous illness; he was well conducted and agreeable; physically, there was an almost complete absence of tremor, and he was much stronger on his legs. This patient is awaiting discharge.

II. Chronic Cases.-Case 12.-This case has been previously described.

Case 13.-Male, aged thirty-five. Admitted August 7, 1923.

Since 1918 he had been irritable and hyperexcitable, and during the last two years his speech had become affected. Trembling had been present since the beginning of his illness.

On examination his general systems showed nothing abnormal. Nervous system: pupils unequal, L. being of the Argyll Robertson variety; tremors of the lips, tongue and face; motor power good, except for trembling in the hands; knee jerks brisk; sphincters normal.

Mentally, the patient was definitely grandiose in his ideas and was very garrulous ; memory poor ; speech markedly affected.

He was inoculated with malaria on August 14, but was only allowed to have five rigors, on account of his condition of prostration. A few weeks after the fever the patient showed slight improvement, and took more interest in his fellow patients, but his speech remained unchanged.

On December 11 this patient came to hospital by himself and appeared to have a fair insight into his condition. His speech had improved very slightly. 
Case 14.-Male, aged forty-six. Admitted June 12, 1923.

General systems appeared normal on examination. Nervous system : pupils unequal and Argyll Robertson in type ; tongue tremulous ; speech thick; knee jerks absent ; plantars flexor ; Rombergism present; Wassermann reaction strongly positive in the blood and cerebrospinal fluid. He was mildly exalted and his conversation foolish ; memory was deficient.

On June 14 he was inoculated with malaria and allowed to have thirteen rises of temperature above $103^{\circ} \mathrm{F}$. During the paroxysms he was querulous and irritable.

Recovery from the anæmia was slow, but ultimately his physical condition reached a much higher level than on admission. His gait was much improved, Rombergism being almost absent. Mentally he was much more alert, but had acquired with his malaria very marked auditory hallucinations, which remained for months after the termination of the fever. His memory was certainly improved.

It is proposed to inoculate this patient again at the end of six months if the improvement is not progressive.

Case 15.-Male, aged sixty. Admitted March 19, 1923.

The general systems appeared normal. Nervous system : pupils equal, with normal reactions; knee jerks brisk; plantars, R. flexor, L. extensor. Mentally he was childish and confused ; memory poor ; delusions ; habits filthy; Wassermann reaction strongly positive in the blood and cerebrospinal fluid.

The patient was inoculated with malaria on July 10. After seven rises of temperature above $103^{\circ} \mathrm{F}$. the attacks ceased spontaneously. He showed no particular reaction during the paroxysms.

Three months later the patient was physically much stronger; his habits were quite reformed and have remained so ever since the termination of the fever.

Case 16. - This case has already been described.

Cases 17 and 18. - These were typical cases of juvenile general paralysis, with the Wassermann reaction strongly positive in the blood and cerebrospinal fluid of both. Both the patients were inoculated with malaria in July, 1923, but after the termination of the fever, their condition remained unchanged.

\section{CONCLUSIONS.}

Our conclusions may be summarized as follows :-

1. In two cases of juvenile paralysis, no improvement was noted. This is in agreement with the findings of Weygandt. ${ }^{15}$

2. Of the chronic cases, five in number :-

One died during the course of the fever.

Four showed some degree of mental and physical improvement; 
one, however, quickly relapsed. In another of these, the auditory hallucinations which were acquired during the course of the fever still persisted after an interval of four months.

3. Of the eleven early cases :-

Two died during the course of the fever.

Three showed very marked mental and physical improvement. Only time will show whether or not these are to be regarded as real ' cures.' It is noteworthy that of these, one became wildly maniacal with each paroxysm, towards the end of the fever, and another, in a similar way, showed very marked confusion. In both cases these symptoms cleared up completely when the fever was terminated.

Three showed considerable improvement, more marked probably on the physical than on the mental side.

One showed a very slight degree of improvement, which has been maintained.

One became decidedly worse after the termination of the fever, and was removed to an asylum.

One showed slight mental improvement, but his physical condition became steadily worse. This patient died four months later from intercurrent disease.

Excluding the two congenital cases, of a series of sixteen cases, three only showed an improvement in any way comparable to the numerous ' cures' of the continental observers. Considering, however, that improvement is stated to be progressive, it is hardly fair to conclude that the cases slightly improved may not ultimately show a complete remission. We are of the opinion that the continental workers are unduly optimistic, but only a widespread trial of the method, over a long period and in a large series of cases, will enable us to confirm or confute their finding. Particularly must the treatment be employed in early cases of the disease. Most of the present series of cases were uncertified patients, admitted to the National Hospital, Queen Square, in a comparatively early stage of the disease. The record of the results obtained in these is, therefore, of the greater value.

We wish to express our thanks to the physicians of the National Hospital, Queen Square, and to the Medical Superintendent, City of London Asylum, Dartford, for their kindness in allowing us to publish the cases.

REFERENCES.

1 Gerstmain, Zeit. f. d. g. Neur. und Psych., 1922, lxxiv, 242.

2 Pilcz, Lancet, 1923, i, 19.

3 GoOdAll, Lancet, 1923, ii, 105.

4 Muhlens and Kirschbaum, Zeit. f. Hyg. und Infek., 1921, xciv, 1.

5 Grant, B.M.J., 1923, ii, 698.

6 WAgner-JacregG, Ars. Med., 1923, i, 40.

7 Templeton, B.M.J., 1923, i, 895. 
8 Gerstmann, Zeit.f. d. g. Neur. und Psych., 1923, Ixxxi, 255.

9 Scripture, Practitioner, 1923, cxi, 367.

10 Quoted by Gerstmann (1).

11 Kirschbaum, Zeit. f. d. g. Neur. und Psych., 1922, lxxv, 635.

12 Robertson, Annual Report, Royal Morningside Asylum, Edinburgh, 1923.

13 McAlister, B.M.J., 1923, ii, 696.

14 Personal communication.

15 Weygand, Klin. Woch., 1923, ii, 2164. 